\title{
Approximating an Auction Mechanism by Multi-Issue Negotiation
}

\author{
Koen Hindriks \\ Delft University of Technology \\ Delft, The Netherlands \\ k.v.hindriks@tudelft.nl
}

\author{
Dmytro Tykhonov \\ Delft University of Technology \\ Delft, The Netherlands \\ d.tykhonov@tudelft.nl
}

\author{
Mathijs de Weerdt \\ Delft University of Technology \\ Delft, The Netherlands \\ M.M.deWeerdt@tudelft.nl
}

\begin{abstract}
The main question addressed in this paper is whether a theoretical outcome determined by an auction mechanism can be reasonably approximated by negotiation among agents in order to drop some of the unrealistic constraints or assumptions presupposed by the mechanism. In particular, we are interested in whether the assumption that a buyer publicly announces her preferences in order to guarantee perfect knowledge of these preferences can be dropped if a negotiating agent is used that can learn preferences. We show how to setup a multiplayer multi-issue negotiation process where preferences are learned, and we investigate how the results of this process relate to the theoretical result of holding an auction in the case of complete knowledge about the preferences of the buyer. Experiments show that the outcomes obtained by negotiating agents that learn opponent preferences approximate the outcome predicted by the mechanism. It thus follows that the assumption of perfect knowledge about buyer preferences can be removed when players are equipped with proper learning capabilities. We also investigate whether the procedure dictated by the mechanism can be further relaxed but in that case experiments indicate that more complex considerations about the market need to be taken into account.
\end{abstract}

\section{INTRODUCTION}

When two parties jointly try to reach an agreement, they follow some kind of negotiation process. When the goal is to make an agreement between one party (the buyer) and one among a number of service providers (sellers), some type of auction can be held. When the agreement involves a number of different issues, for example on the criteria and constraints of a service, a multi-attribute auction is suitable. However, such auctions have several requirements. The most important one being that the sellers must have perfect knowledge about the preferences of the buyer.

If the service request is completely specified, this assumption is easily satisfied (cheaper is better), but often the buyer can have complex preferences over a set of issues. In most such settings it is unrealistic to assume that service providers have perfect knowledge about the preferences of the buyer. This invalidates the use of an auction. However, through negotiation beliefs about the preferences of a buyer can be formed. This led us to the question whether we could achieve a similar outcome by using some kind of multiplayer multi-issue negotiation process, where the sellers learn the preferences of the buyer during this process.

In the next section we define a general multi-issue problem, which also captures the setting of selecting a service from one of a set of potential service providers. Then we briefly describe an auctionlike approach that can be used in a setting where the buyer is able to announce its preferences to all sellers. For situations where this is undesirable, we propose a multi-player negotiation method to approximate the outcome of this mechanism in Section 4. The outcomes of this method are then experimentally compared to the outcomes of the auction mechanism in Section 5. The paper is concluded in Section 6 with a discussion of the results, some related work, and directions for future research.

\section{PROBLEM DEFINITION}

In a setting where a buyer is interested in obtaining a service from one of a set of service providers, an outcome is an agreement $x=\left\langle x_{1}, \ldots, x_{m}\right\rangle \in X$ over all $m$ issues in a domain $X=X_{1} \times$ $\ldots \times X_{m}$. These issues define all aspects of the agreement, such as price, quality, start time, duration, guarantees, penalty, etc. With each agreement both the buyer and each service provider (seller) associate a utility value.

We introduce the following notation. Sellers are denoted by $i \in\{1, \ldots, n\}$, the buyer is denoted by 0 . Each player $i$ has a reservation value $v_{i}$ which indicates the smallest value an agreement should have to be acceptable to the player, and a utility function $u_{i}: X \rightarrow \mathbb{R}$ which defines the utility of a certain agreement to player $i$.

The goal is to find an agreement between the buyer and one of the sellers that is not only acceptable to both, but that is also Paretooptimal, i.e., there should not be another agreement with the same or higher utility for both players, and strictly higher for at least one of them. Furthermore, if possible, we would like to deal with the issue of fairness. In two player negotiations, fairness can be defined formally by for example the Nash or Kalai-Smorodinsky solution (see e.g., Thomson, 1994). However, in this setting with one buyer and multiple sellers, the buyer has in general more negotiation power than the sellers, and agreements therefore may be expected to deviate from these particular solution concepts. In the next section a mechanism is introduced that has a dominant strategy equilibrium that yields a Pareto efficient outcome, provided there are two or more sellers.

\section{AUCTION MECHANISM}

Earlier work has shown how to reach an agreement between a buyer and one of a set of sellers using a closed-bid auction-like mechanism (Harrenstein et al., 2008). Intuitively, this mechanism captures the negotiation power of the buyer. If there are many sellers, the buyer will end up with some very good offers, but if there is only one seller that has a sufficiently good offer, the agreement is not that good for the buyer. This interpretation can be given to most auction mechanisms. The mechanism described below has the special feature that it also works if none of the issues is about money. (If money is involved, the relative value of alternatives for other issues may be assigned a price, i.e. be translated into money.) The mechanism can be thought of as consisting of two rounds. In 
the first round (1a-c), the buyer publicly announces her preferences, potential service providers (sellers) submit offers in response, and a winner is selected by the buyer. The winner selected is the seller who has submitted the best offer from the point of view of the buyer. After establishing the winner, in a second round (2a-b), the buyer determines the second-best offer (from her perspective again) she received from another seller, announces this publicly, and then the winner is allowed to select any agreement that has at least the same utility to the buyer as the second-best offer (which can be determined by the winner since the preferences of the buyer are publicly announced). We assume the bids offered in the first round all go through a trusted third party, such as a sollicitor, who can check whether the buyer keeps the protocol. Summarizing, the steps of the procedure are:

1a. The buyer announces her preferences.

1b. Every seller submits an offer.

1c. The buyer selects the winner according to her preferences.

2a. The buyer announces the second-best offer she received.

2b. The winner may select any agreement that has at least the same utility for the buyer as the second-best offer.

One of the nice properties of this mechanism is not only that the seller wins that can make the best offer, but also that it is a dominant strategy for a seller to bid an offer that is acceptable to itself and ranks highest in the buyer's preferences. An offer is acceptable to a seller if her utility is at least the reservation value. In the problem domain defined in the previous section, this dominant strategy comes down to proposing an offer with exactly the same utility as its reservation value. Formally, the winner in a given problem domain $X$ is defined by:

$$
i^{*}=\arg \max _{i \in\{1, \ldots, n\}} \max \left\{u_{0}(x) \mid x \in X, u_{i}(x) \geq v_{i}\right\} .{ }^{1}
$$

where $v_{i}$ denotes the reservation value of seller $i$.

To determine the outcome, we also need the second-best offer. Again, assuming all sellers follow the dominant strategy, the second-best offer $\hat{x}$ is given by

$$
\hat{x}=\arg \max _{x \in\left\{x \mid u_{i}(x) \geq v_{i}, i \in\{1, \ldots, n\} \backslash\left\{i^{*}\right\}\right\}} u_{0}(x) .
$$

The outcome then is the best possible for the winner $i^{*}$, given that it is at least as good for the buyer as the second-best offer $\hat{x}$.

$$
\omega=\arg \max _{x \in\left\{x \mid u_{0}(x) \geq u_{0}(\hat{x})\right\}} u_{i^{*}}(x) .
$$

This outcome of the auction-like mechanism is Pareto-efficient, because in the last step the winner maximizes its utility given a constraint on the utility for the buyer (and full knowledge of both preferences). Note that in this paper we assume that the utility functions of the buyer and the sellers are not completely aligned. Therefore, the best outcome for the winner actually lies on the boundary defined by the constraint on the utility for the buyer (i.e., $u_{0}(\omega)=u_{0}(\hat{x})$ ). Figure 1 illustrates this outcome using the Pareto fronts of the buyer with the winner and the buyer with the secondbest seller. Two different figures are provided to illustrate that the outcome for the buyer depends on the available offers of sellers, i.e. the seller market. In a market where few sellers are available the second-best outcome from the buyer's perspective may not be as good as a market where many sellers compete and a buyer may hope for a better outcome (compare bottom figure with top figure in Figure 1).

\footnotetext{
${ }^{1}$ We assume ties are broken by the buyer using a given ordering over the sellers.
}
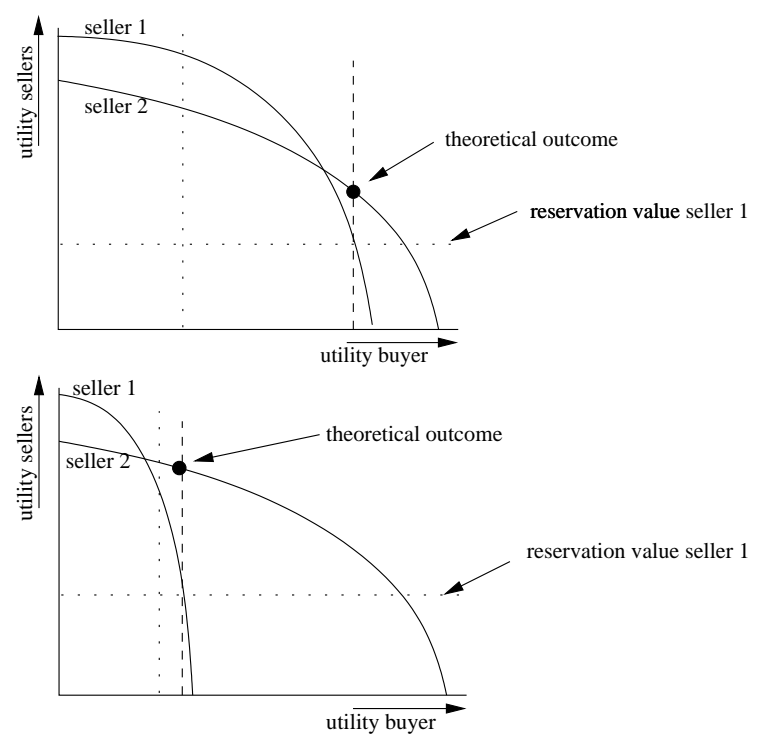

Figure 1: The theoretical outcome for the winner depends on the highest acceptable offer of the second-best seller.

To see that it is a dominant strategy for the sellers to bid their highest acceptable offer, consider the following two cases for a seller $i$. Let $x$ denote $i$ 's highest-ranked acceptable offer, and $\hat{x}$ the highest ranked offer over all other players. If $\hat{x}>x$, then $i$ should bid below $\hat{x}$ according to the buyer's preferences, because if she wins, she can only select unacceptable outcomes. If $x>\hat{x}$, then $i$ should bid above $\hat{x}$ in the buyer's preferences, because then her outcome is highest and she will be the winner. In both cases, the optimal strategy for $i$ is to offer $x$.

This mechanism is similar to the one described by Harrenstein et al. (2008) except for the model of the preferences of the players. In this paper we assume that every player assigns a utility to each possible outcome, while in the original work the only assumption is that every player has an ordering over outcomes.

The main problem with a realistic implementation of the mechanism described above is that the buyer needs to communicate her preferences to all sellers. This is impractical for various reasons. Firstly, in many settings it is undesirable for the buyer to communicate all her preferences to all sellers, because the buyer may not want to disclose all details for strategic reasons. Secondly, this preference function can be quite a complex function over a large domain, which is difficult to communicate efficiently. Finally, a buyer may not even know the complete domain of agreements on forehand, even though she is able to rank any given subset of agreements. The latter holds for example when a government sends out a request for proposals to construct a bridge over a river within a given budget. It is impossible to list all possible types of bridges designers may come up with. In the next section we describe an approach based on negotiation that may be used to approximate the mechanism and where there is no need to publicly announce the preferences of the buyer.

\section{APPROXIMATION BY NEGOTIATION}

An alternative available to sellers that need to make offers to a buyer that does not publicly announce her preferences is to learn these preferences during a negotiation session. Here we use a Bayesian technique for learning opponent preferences in single-shot negotiations (Hindriks and Tykhonov, 2008a). By using this learning ca- 
pability, it is possible to implement a negotiation setup that mimics the steps to compute the mechanism outcome in which the preferences of the buyer need not be revealed. The negotiation is structured in two rounds to match the structure of the mechanism. In the first round negotiation sessions are performed between the buyer and every potential seller. An alternating offers protocol is used in each of the negotiations. Moreover, the negotiation sessions are assumed to be independent. At the end of the first round, a winner (one of the sellers) is determined. Before starting the second round, the agreement between the seller and buyer that is second-best from the perspective of the buyer is revealed to all sellers, in particular to the winner. In the second round an agreement between the winner and the buyer is established.

We discuss two different negotiation setups that we have experimented with in order to verify how closely they approximate the mechanism outcome. In both setups the buyer will not reveal her preferences. The idea is that in the first round agents try to learn these preferences. In the first setup we discuss, the agents are required to use a negotiation procedure that implements the steps of the mechanism otherwise quite closely. That is, in the first round all offers proposed by sellers are required to have a utility equal to their reservation value. This constraint is derived from the fact that the dominant strategy for sellers is to propose such offers given the mechanism discussed in the previous section. In the second round all offers proposed by the buyer are required to have a utility equal to that of the second-best outcome of the first round. Again, this constraint is derived from the rule to compute the final outcome of the mechanism. The intuition is that the buyer knows it can have a deal with another seller. It informs the winner of this alternative to reduce the negotiation space. An alternative way of putting this is that the winner of the first round is required to adjust its reservation value and increase it to the utility it associates with the second-best outcome as revealed by the buyer (if that outcome has a higher utility than its initial reservation value; otherwise, the seller would not change its reservation value and the other sellers would have done a very bad job negotiating against the buyer). Given that utilities are either fixed for the buyer or the seller, it is rational for these agents to try to propose the best deal possible for the other party. In general this is the case since negotiators need to take into account that an offer needs to be reasonable for the other party in order to reach an agreement at all. More specifically, for sellers, in the first round, this is also the case because they need to win in this round to go through to the second. Given this setup, our hypothesis about the feasibility to approximate the mechanism outcome by means of negotiation is the following.

HYPOTHESIS 1. The outcome determined by the mechanism can be approximated by a negotiation setup in which: (i) the buyer does not reveal her preferences, (ii) the negotiating agents can learn an opponent's preference profile, and (iii) these agents use the negotiation procedure discussed above.

Note that in the first round, the buyer is free to chose the offers she proposes. This makes it possible for the seller to learn the preferences the buyer has during this negotiation. Also note that as the seller is supposed to propose offers with a fixed utility value (equal to its reservation value) it is difficult if not impossible for the buyer to learn the preferences of sellers in this round. Figure 2 illustrates the first setup and the constraints on the negotiation moves of the sellers in the first round and the buyer in the second round.

In the first setup, we only drop the assumption that the buyer reveals its true preferences to all sellers. It is natural to ask whether we can further relax the procedure, i.e. the constraints on the strategies that are imposed on the negotiating agents in the first setup.
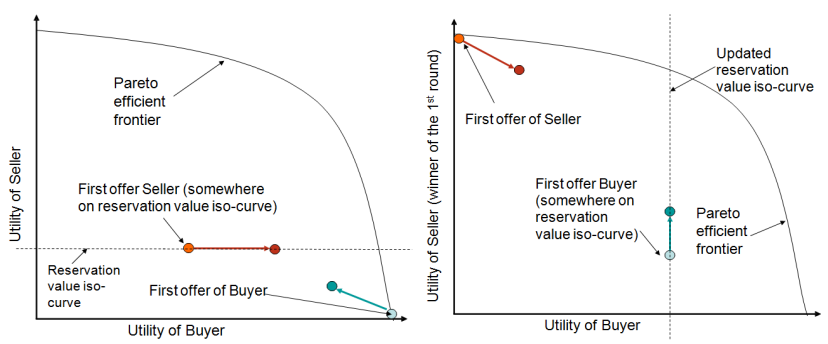

Figure 2: Negotiation Moves in the First Setup
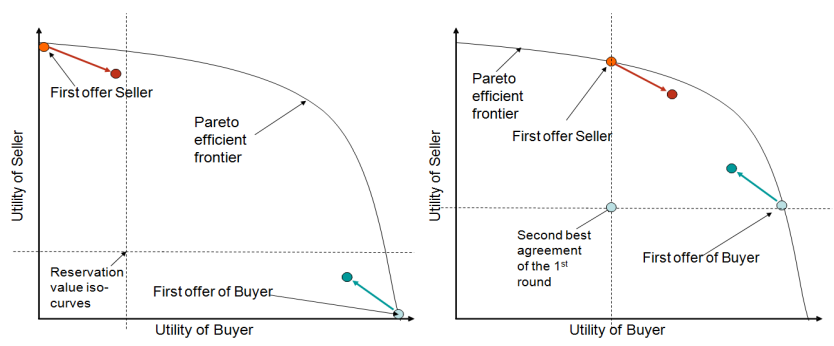

Figure 3: Negotiation Moves in the Second Setup

In the second setup we have investigated, we additionally drop the assumption that the seller in the first round and the buyer in the second round have to propose offers with a utility value that is fixed. Effectively, this means that a closed bilateral negotiation as any other negotiation is started in the first round where both players may learn from this round about the preferences of the other party. In particular, there are no constraints on the strategy used by either of the negotiating parties, although in the experiments we used the agents proposed by Hindriks and Tykhonov (2008a).

Apart from this, the basic structure of the negotiation is kept, and a second round is started after the second-best outcome of the first round has been made public by the buyer. The winner of the first round negotiates with the buyer to reach a final agreement in this round. The effect of making the second-best outcome public is modeled by having both players adjust their reservation values given the utilities they associate with it (of course, the seller would only do this if it improves its current reservation value). In line with these adjustments and the public announcement of the second-best outcome, negotiation in the second round moreover starts with different offers than in the first round (see figure on the right in Figure 3). It is assumed that both players start with an offer that has a utility equal to the reservation value of its opponent and lies on the Pareto curve. This offer by necessity is only an approximation of this point as it has to be determined by means of the learned opponent model which was learned in the first round. This second round negotiation therefore depends also on the quality of the learning mechanism.

\section{EXPERIMENTAL EVALUATION}

In this section, we first discuss the design of the experiment. We then present the experimental results of the first and second setup and discuss our findings. The results for the first setup are in line with the hypothesis formulated in Section 4. The results obtained for the second setup, however, show major deviations from the winner and outcomes as determined by the mechanism, and we discuss why this is the case. 


\subsection{Experimental Design}

The original theoretical mechanism does not limit the number of sellers in the auction. To create an experimental setup the negotiation mechanism must be able to select a winner and the secondbest offer. Therefore, only a setup with two sellers is sufficent to test the proposed approach. The setup can easily be generalized to any number of sellers. Observe that given any two sellers in the proposed negotiation setup the addition of a new one would not decrease the buyer's utility. If the newcomer has a preference profile that does not suit the buyer's needs it would neither be selected as a winner nor used as a second-best offer. On the other hand, if it would better satisfy the buyer's need it would only increase the competition among the sellers. In the latter case, the sellers competition would strengthen the buyer's position and, as a result, lead to a better outcome for the buyer.

In the experiments, we use the so-called Service-Oriented Negotiation domain, taken from Faratin et al. (2003). This domain consists of 4 issues. These issues are supposed to represent various attributes important and relevant with respect to a service being offered, such as price, quality, time, and penalty. Here, we do not assume that any of these issues are predictable, i.e. it is not possible to make reasonable estimates about the preferences by an opponent before negotiation is started. This means that we have more variation in the profiles we used in the experiments than one would typically expect in this domain. As a consequence, our results are more easily generalized to other settings. A range of profiles were used with different reservation values, importance weights associated with issues, and evaluation of alternatives associated with each issue. More specifically, a set of 12 preference profiles per role are created for the experimental setup. The following values for each of the parameters were used:

1. To model the relative importance of the value of the issues, two different sets of weights are used. One representing equal importance of all issues, using 0.25 as weight for each of the four issues, and a set of weights representing dominance of two issues over the other two, using the weights $0.30,0.50,0.05$, and 0.15 .

2. The reservation value of each agent is set to either 0.3 or 0.6 .

3. The relative value associated with each of alternatives for an issue is represented by a linear "uphill" function, a linear "downhill" function, or a combination of the two (resulting in a triangular shape). That is, three types of evaluation functions were used to assign relative value to issue alternatives.

Figure 4 shows an example of a preference profile for a buyer. For presentation purposes, utility function of an individual issue on the figure is mutiplied by the corresponding weight. Utility of a complete bid can be calculated by summation of the utilities of individual issues.

Using these parameter values, a sample of 50 different negotiation setups is created by means of random selection of one of the twelve profiles for each role. Moreover, as a seller with a lower reservation value in such a setup has a higher chance of winning the first round (due to convexity of the Pareto efficient frontier), the sample is balanced such that in $80 \%$ of the cases the sellers have equal reservation values.

In the experiments, the negotiating agents build a model of the opponent preferences, by means of learning a probability distribution over a set of hypotheses about the utility function (i.e. the evaluation functions and weights of issues) of the opponent (Hindriks and Tykhonov, 2008a). The probability distribution is defined over
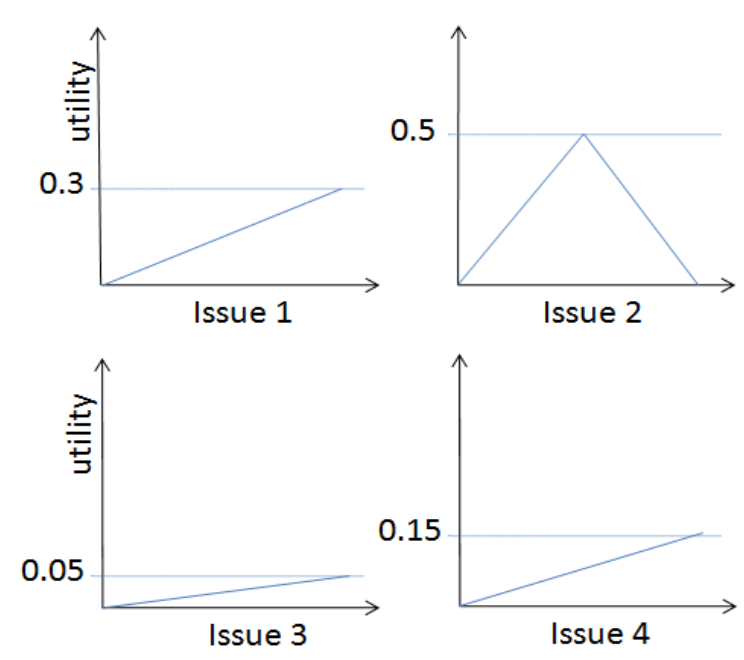

Figure 4: Example of a preference profile of a buyer with weights $0.30,0.50,0.05$, and 0.15 . Issues 1,3 , and 4 have "uphill" utility function, issue 2 has a "triangular" shape utility function.

the set of hypotheses that represent an agent's beliefs about an opponent's preferences. Structural assumptions about the evaluation functions and weights are made to decrease the number of parameters to be learned and simplify the learning task. Each hypothesis represents a complete utility space as a combination of weights ranking and shapes of the issue evaluation functions.

During a negotiation every time when a new bid is received from the opponent the probability of each hypothesis is updated using Bayes' rule. This requires a conditional probability that represents the probability that the bid might have been proposed given a hypothesis. Therefore the utility of the bid is calculated according to this hypothesis and compared with the predicted utility according to the assumption about rationality of the opponent's negotiation tactics. To estimate the predicted utility value an assumption about the opponent concession tactics is used based on a linear function. The linear function is the most plausible model of the negotiation concession tactic used by the opponent.

\subsection{First Setup}

The results for the first set of experiments validate our hypothesis that negotiating agents that can learn are able to approximate the outcome determined by the mechanism quite well. In Figure 5, a subset of the outcomes are presented to illustrate some of the results obtained (we selected 13 results out of a total of 48 experiments we performed to provide a good, representative sample). In the left column labelled Theoretical Results the winners of the first round and the outcomes of the second round as determined by the mechanism are listed. In the right column labelled Experimental Results the winners of the first round and the resulting outcomes of the second round as obtained by the negotiating agents are presented. The difference as a percentage of the buyer and seller outcomes respectively are listed last in each row.

First, note that the winner predicted by the mechanism and the negotiation setup coincide $100 \%$. This means that the negotiation setup does not change the results of the first round in which a seller is selected as winner. Moreover, in the second round, in general the outcomes obtained by negotiation are also quite close to those determined by the mechanism. That is, in $78 \%$ of the experiments the 


\begin{tabular}{|c|c|c|c|c|c|c|c|}
\hline \multicolumn{3}{|c|}{ Theoretic Results } & \multicolumn{5}{|c|}{ Experimental Results } \\
\hline \multirow[b]{2}{*}{ Winner } & \multicolumn{2}{|c|}{ Final agreement } & \multirow[b]{2}{*}{ Winner } & \multicolumn{2}{|c|}{ Final agreement } & \multicolumn{2}{|c|}{ Difference } \\
\hline & Buyer & Seller & & Buyer & Seller & Buyer & Seller \\
\hline Seller A & 0.81 & 0.60 & Seller A & 0.81 & 0.63 & $-1 \%$ & $4 \%$ \\
\hline Seller B & 0.67 & 0.91 & Seller B & 0.64 & 0.88 & $-5 \%$ & $-4 \%$ \\
\hline Seller A & 0.66 & 0.73 & Seller A & 0.66 & 0.74 & $-1 \%$ & $2 \%$ \\
\hline Seller B & 0.67 & 0.60 & Seller B & 0.60 & 0.65 & $-11 \%$ & $8 \%$ \\
\hline Seller A & 0.98 & 0.36 & Seller A & 0.94 & 0.45 & $-4 \%$ & $26 \%$ \\
\hline Seller B & 0.67 & 0.60 & Seller B & 0.60 & 0.66 & $-11 \%$ & $11 \%$ \\
\hline Seller B & 0.85 & 0.41 & Seller B & 0.77 & 0.37 & $-10 \%$ & $-10 \%$ \\
\hline Seller B & 0.85 & 0.60 & Seller B & 0.79 & 0.65 & $-7 \%$ & $7 \%$ \\
\hline Seller B & 0.86 & 0.39 & Seller B & 0.85 & 0.40 & $-1 \%$ & $3 \%$ \\
\hline Seller B & 0.85 & 0.41 & Seller B & 0.77 & 0.46 & $-10 \%$ & $12 \%$ \\
\hline Seller A & 0.70 & 0.56 & Seller A & 0.71 & 0.56 & $1 \%$ & $0 \%$ \\
\hline Seller B & 0.68 & 0.59 & Seller B & 0.67 & 0.59 & $-1 \%$ & $0 \%$ \\
\hline Seller B & 0.71 & 0.83 & Seller B & 0.71 & 0.83 & $0 \%$ & $0 \%$ \\
\hline Seller B & 0.89 & 0.55 & Seller B & 0.79 & 0.67 & $-11 \%$ & $21 \%$ \\
\hline
\end{tabular}

Figure 5: Example Results Obtained in the First Setup

\begin{tabular}{|c|c|c|c|c|c|c|c|}
\hline \multicolumn{3}{|c|}{ Theoretic Results } & \multicolumn{5}{|c|}{ Experimental Results } \\
\hline \multirow[b]{2}{*}{ Winner } & \multicolumn{2}{|c|}{ Final agreement } & \multirow[b]{2}{*}{ Winner } & \multicolumn{2}{|c|}{ Final agreement } & \multicolumn{2}{|c|}{ Defference } \\
\hline & Buyer & Seller & & Buyer & Seller & Buyer & Seller \\
\hline Seller A & 0.81 & 0.60 & Seller A & 0.73 & 0.63 & $-10 \%$ & $4 \%$ \\
\hline Seller B & 0.67 & 0.91 & Seller B & 0.82 & 0.63 & $22 \%$ & $-31 \%$ \\
\hline Seller A & 0.66 & 0.73 & Seller A & 0.71 & 0.52 & $8 \%$ & $-28 \%$ \\
\hline Seller B & 0.67 & 0.60 & Seller A & 0.62 & 0.56 & $-8 \%$ & $-7 \%$ \\
\hline Seller A & 0.98 & 0.36 & Seller B & 0.78 & 0.76 & $-21 \%$ & $112 \%$ \\
\hline Seller B & 0.67 & 0.60 & Seller B & 0.70 & 0.55 & $3 \%$ & $-8 \%$ \\
\hline Seller B & 0.85 & 0.41 & Seller B & 0.67 & 0.58 & $-22 \%$ & $40 \%$ \\
\hline Seller B & 0.85 & 0.60 & Seller B & 0.81 & 0.63 & $-5 \%$ & $5 \%$ \\
\hline Seller B & 0.86 & 0.39 & Seller A & 0.76 & 0.53 & $-12 \%$ & $35 \%$ \\
\hline Seller B & 0.85 & 0.41 & Seller B & 0.70 & 0.55 & $-18 \%$ & $33 \%$ \\
\hline Seller A & 0.70 & 0.56 & Seller A & 0.65 & 0.56 & $-7 \%$ & $0 \%$ \\
\hline Seller B & 0.68 & 0.59 & Seller A & 0.69 & 0.59 & $1 \%$ & $0 \%$ \\
\hline Seller B & 0.71 & 0.83 & Seller B & 0.68 & 0.85 & $-4 \%$ & $2 \%$ \\
\hline Seller B & 0.89 & 0.55 & Seller A & 0.86 & 0.65 & $-3 \%$ & $18 \%$ \\
\hline
\end{tabular}

Figure 6: Example Results Obtained in the Second Setup deviation is less than $5 \%$. The standard deviation of the difference between the mechanism outcome and the experimental results is $4 \%$, and in $94 \%$ of the experiment the deviation did not differ with more than $10 \%$, indicating that overall outcomes were reasonably close to the mechanism outcome with a few exceptions.

The Bayesian learning agent has been shown to be capable of learning various preference profiles (Hindriks and Tykhonov, 2008b). However, a decay of the buyer's utility was observed in the cases where its preference profile had the $\langle 0.30 ; 0.50 ; 0.05 ; 0.15\rangle$ vector of weights. This can be explained by the fact that the Bayesian learning agents use only linear ranking of the issues in the hypotheses and the closest one would be $\langle 0.30 ; 0.40 ; 0.20 ; 0.10\rangle$. The difference in the absolute values of the weights results in less perfect learning in these cases. A drop in the utility of the buyer was usually compensated by an increase of the seller's utility. The utility of the buyer is in fact determined in the first round of negotiations by the quality of the second-best offer. Therefore, it is in the interest of the buyer to allow the potential sellers to learn its preference profile and be at least somewhat transparent.

In this setup all agents try to maximize opponent's utility when following the negotiation strategy using the learned model of opponent preferences. Therefore, selection of the winner as well as the second-best offer depends on abililty of a buyer to learn preference profile of the seller. Furthermore, utility of the winning buyer in the final agreement is determined by the seller's ability to learnit the buyer's preference profile. From this we conclude that in this setup ability of an agent to learn opponent prferences is a key factor in a successful approximation of the auction mechanism.

As predicted in the previous section a low reservation value of the seller increases chances of winning the first round of negotiations. Indeed, as the results show, in 8 out of 10 setups where sellers had different reservation values the seller with the lowest reservation value won the first round.

\subsection{Second Setup}

Results of the second setup show much stronger deviation of the reached negotiation outcomes from the theoretical results. Figure 6 presents the results obtained in the second setup. In 33\% of the experiments the winner was not the same as the predicted one. The standard deviation of the absolute difference between the theoretical and the experimental results is now $12 \%$. Because this approximation setup was tested in the same experimental setup the learning performance was as good as in the first setup and the negotiation strategy is able to find agreements close to the Pareto efficient frontier. This is supported by the fact that a decrease in the buyer's utility was compensated by a increase in the seller's utility of an agreement. As such, we conclude that other factors must explain the deviation of the results obtained in this setup. In particular, dropping the constraints on the strategies of both buyer and seller in the way proposed in this setup decreases the power of the buyer significantly (as can also be concluded from some of the dramatic improvements sellers reached in some of the experiments). The setup does not reflect the fact that sellers need to take into account that they are in competition with other sellers to be selected as service provided. Basically, the assumption that negotiations are independent is invalid in this context and sellers need to adapt their strategy to take other competitors explicitly into account.

One idea to improve this setup in order to obtain a better approximation of the mechanism outcome is to use estimates of the reservation value of other sellers to select negotiation moves, i.e. to adapt one's negotiation strategy. As we saw in the previous section, the reservation value of sellers plays a significant role in determining the winner and, consequently, the buyer's outcome. A modified negotiation setup could take possible values of the reservation values of other sellers into account when selecting an offer.

\section{CONCLUSION}

The work in this paper is a first step towards dealing with the main undesirable feature of an auction mechanism, being that the preferences of the buyer need to be publicly known.

The first experiment shows that given certain restrictions on the negotiation strategies, it is possible to approximate the outcome of such an auction mechanism by multiplayer negotiations, and thus this eliminates the need to publish the preferences of the buyer. The results in this setup could not have been so good if the learned models had been far from correct. An incorrectly learned model explains the results where the agreement diverted from the mechanism outcome.

The results of the second experiment indicate that independent negotiations cannot be used in a setting where one buyer interacts with multiple sellers. Sellers should take into account that other sellers may outbid them, and therefore follow a different strategy, which is dependent on (the expectation of) the behavior of other sellers. The first experiment can be seen as an extreme way of dealing with this, by following the strategy of staying near your reservation value.

Other work has dealt with this problem only from the buyer's side. Rahwan et al. (2002) and later Nguyen and Jennings (2003) proposed a negotiation framework where the buyer negotiates with a number of sellers concurrently, and updates its reservation value 
in all other negotiation threads with the value of an agreement, whenever one is made. The latter work presents experimental results on the effect of a number of negotiation strategies in a setting where each utility function is a standard linear combination of the issues. It seems that in such a parallel setting the speed of the negotiation threads may influence the changes in reservation value of the buyer and thus the result. The effect of this has not yet been studied, but if this leads to undesirable results, it may be interesting to try an approach in two rounds, like the one proposed in our work here.

Another line of work in this field includes an expectation about results obtained in other threads ( $\mathrm{Li}$ et al., 2004). Like in the work discussed above, the reservation value for the buyer is set based on events in the other threads. The interesting extension here is that the reservation value can be set at the expected best offer in other threads, or even in future threads. Though the negotiations in this work are only about price, it seems that this idea could be generalized to a setting with multiple issues as well.

An empirical study of comparing an auction mechanism with a negotiation mechanism was conducted by Kjerstad (2005). The aim of this study is to estimate the impact of trading mechanisms on the price of an agreement. The paper considers data of 216 trades that result from either using an auction or a negotiation as the trade mechanim. It is concluded that trade mechanisms can have an influence on the number of suppliers due to, i.e., costs associated with a particular mechanism. The results show that the choice of the trading mechanism does not influence the price of an agreement, however.

An interesting direction for future research is to study the role of the sellers market in our setting. As the results showed, the preference profile and the reservation value of a seller have a strong influence on the buyer's utility of the outcome. Increase in the number of sellers in the setup would result in an increase of the competition among the sellers. In the current setup, we assumed independence of negotiations in the first round. It would be interesting to see how the strategy of the sellers would need to change if they become aware of each other and how this would influence the outcome, in particular whether it would provide for a better approximation of the auction mechanism discussed here.

\section{ACKNOWLEDGEMENTS}

Dmytro Tykhonov and Mathijs de Weerdt are supported by the Technology Foundation STW, applied science division of NWO, and the Ministry of Economic Affairs of the Netherlands.

\section{References}

Faratin, P., Sierra, C., and Jennings, N. R. (2003). Using similarity criteria to make negotiation trade-offs. Journal of Artificial Intelligence, 142(2):205-237.

Harrenstein, P., Mahr, T., and de Weerdt, M. M. (2008). A qualitative vickrey auction. In Endriss, U. and Paul W, G., editors, Proceedings of the 2nd International Workshop on Computational Social Choice, pages 289-301. University of Liverpool.

Hindriks, K. and Tykhonov, D. (2008a). Opponent modelling in automated multi-issue negotiation using bayesian learning. In Proceedings of the 7th International Conference on Autonomous Agents and Multiagent Systems (AAMAS'08), pages 331-338.

Hindriks, K. and Tykhonov, D. (2008b). Towards a quality assessment method for learning preference profiles in negotiation. In Proceeding of Workshop on Agent-based Complex Automated Negotiations, pages 64-71.
Kjerstad, E. (2005). Auctions vs negotiations: A study of price differentials. Econometrics and Health Economics, 14:12391251.

Li, C., Giampapa, J., and Sycara, K. (2004). Bilateral negotiation decisions with uncertain dynamic outside options. In Proc. of 1 st IEEE Int. Workshop on Electronic Contracting, pages 54-61.

Nguyen, T. D. and Jennings, N. (2003). Concurrent bilateral negotiation in agent systems. In Proc. of 14th Int. Workshop on Database and Expert Systems Applications, pages 844-849.

Rahwan, I., Kowalczyk, R., and Pham, H. (2002). Intelligent agents for automated one-to-many e-commerce negotiation. In Proc. of 25th Australasian Conf. on Computer Science, pages 197-204. Australian Computer Society, Inc., Darlinghurst, Australia.

Thomson, W. (1994). Cooperative models of bargaining. In Aumann, R. and Hart, S., editors, Handbook of Game Theory, Volume 2, chapter 35. Elsevier Science B.V. 